\title{
SOME REDUCED FREE PRODUCTS OF ABELIAN $C^{*}$-ALGEBRAS AND SOME $C^{*}$-SUBALGEBRA IN A FREE PRODUCT
}

\author{
Jaeseong Heo And JeOng Hee Kim
}

\begin{abstract}
We prove that the reduced free product of $k \times k$ matrix algebras over abelian $C^{*}$-algebras is not the minimal tensor product of reduced free products of $k \times k$ matrix algebras over abelian $C^{*}$-algebras. It is shown that the reduced group $C^{*}$-algebra associated with a group having the property $T$ of Kazhdan is not isomorphic to a reduced free product of abelian $C^{*}$-algebras or the minimal tensor product of such reduced free products. The infinite tensor product of reduced free products of abelian $C^{*}$-algebras is not isomorphic to the tensor product of a nuclear $C^{*}$-algebra and a reduced free product of abelian $C^{*}$-algebra. We discuss the freeness of free product $\mathrm{II}_{1}$-factors and solidity of free product $\mathrm{II}_{1}$-factors weaker than that of Ozawa. We show that the freeness in a free product is related to the existence of Cartan subalgebras in free product $\mathrm{II}_{1}$-factors. Finally, we give a free product factor which is not solid in the weak sense.
\end{abstract}

\section{Introduction}

The study of the free probability theory has seen rapid and impressive progress since it was introduced by Voiculescu in the framework of operator algebras. Free probability theory has turned out to be very powerful in the study of von Neumann algebras associated with free products of discrete groups, in particular, of free group factors. In the non-commutative probability theory there is a notion of the free product of finite von Neumann algebras with specified traces, for which one has $L\left(G_{1}\right) * L\left(G_{2}\right) \simeq L\left(G_{1} * G_{2}\right)$ where $L(G)$ is the finite von Neumann algebra associated to a discrete group $G$. In particular, the reduced free product of $C^{*}$-algebras is closely related to the free product of groups via the reduced group $C^{*}$-algebras. The notion of freeness in operator algebras can be viewed as an abstract extension of freeness in groups.

Received March 25, 2009

2000 Mathematics Subject Classification. Primary 46L09, Secondary 46L06.

Key words and phrases. free product of $C^{*}$-algebras, Powers' group, minimal tensor product, stable rank 1, prime factor, property $T$, Cartan subalgebra.

This work was supported by a grant No. R01-2007-000-20064-0 from the Korea Science and Engineering Foundation (KOSEF). 
There are many questions about (reduced) free products. One of most basic questions concerns simplicity of free product $C^{*}$-algebras or $C^{*}$-subalgebras of free product von Neumann algebras. Powers [12] showed that the reduced group $C^{*}$-algebra $C_{r}^{*}(G)$ is simple and has a unique trace when $G$ is the free group $\mathbb{F}_{2}$ on two generators. This example intrigued the further deep structure theory for $C^{*}$-algebras and notably $K$-theory for $C^{*}$-algebras. Furthermore, his method turned out to be a prototype for inferring the simplicity of $C^{*}$-algebras. Avitzour [1] introduced a free product of $C^{*}$-algebras with faithful states and generalized Powers' result to free products of $C^{*}$-algebras. There are also many questions concerning the basic structure of factors, in particular, of free group factors. It has recently proved by Ozawa and Popa [10] that $\bigotimes_{k=1}^{n} L\left(\mathbb{F}_{2}\right)$ is not *-isomorphic to $\bigotimes_{k=1}^{m} L\left(\mathbb{F}_{2}\right)$ if $n \neq m$. We will here consider the $C^{*}$-algebraic analogue of this result. We refer the survey paper [14] for a detail discussion, some properties of operator algebras associated to the free groups and related geometric groups giving rise to algebras with similar properties. See $[16,8]$ for more detailed information of free products of $C^{*}$-algebras.

In this paper we concern with the simplicity, primality and freeness of (reduced) free products. We first show that the reduced free product of $k \times k$ matrix algebras over abelian $C^{*}$-algebras is not the minimal tensor product of reduced free products of $k \times k$ matrix algebras over abelian $C^{*}$-algebras and that they are exact and have stable rank 1 . It is shown that the reduced group $C^{*}$-algebra associated with a group having the Kazhdan's property $T$ is not isomorphic to a reduced free product of abelian $C^{*}$-algebras or the minimal tensor product of such reduced free products. Furthermore, the infinite tensor product of reduced free products of abelian $C^{*}$-algebras is not isomorphic to the tensor product of a nuclear $C^{*}$-algebra and a reduced free product of abelian $C^{*}$-algebra. In the third section, we show the simplicity of the $C^{*}$-algebra generated by some $C^{*}$-subalgebra and a Haar unitary in a free product of finite von Neumann algebras and the uniqueness of a trace. This result has already been proved by Dykema [4], but we are showing by the different method extending directly the Powers' technique. We discuss the freeness of free product $\mathrm{II}_{1}$-factors and solidity of free product $\mathrm{II}_{1}$-factors weaker than that of Ozawa [9]. We show that the freeness in a free product is related to the existence of Cartan subalgebras in free product $\mathrm{II}_{1}$-factors. Finally, we give a free product factor which is not solid in the weak sense.

\section{On reduced free products of abelian $C^{*}$-algebras}

In this section we investigate the simplicity and stable rank of free products of abelian $C^{*}$-algebras. We first would like to remind the definition of reduced free products of $C^{*}$-algebras [15]. Let $I$ be an index set and let $A_{\iota}(\iota \in I)$ be a unital $C^{*}$-algebra with a state $\phi_{\iota}$ whose associated GNS representation is faithful. The reduced free product of $\left\{A_{\iota}, \phi_{\iota}: \iota \in I\right\}$ is the (unique) unital $C^{*}$-algebra $(A, \phi)$ with unital embeddings $\mathcal{A}_{\iota} \hookrightarrow A$ such that 
(i) the GNS representation associated with $\phi$ is faithful on $A$;

(ii) $\left.\phi\right|_{\mathcal{A}_{\iota}}=\phi_{\iota}$;

(iii) $\left\{A_{\iota}: \iota \in I\right\}$ is free with respect to $\phi$;

(iv) $A$ is generated by $\left\{A_{\iota}: \iota \in I\right\}$.

We denote the reduced free product by $(A, \phi)=*_{\iota \in I}\left(A_{\iota}, \phi_{\iota}\right)$. It is known that $\phi$ is a trace if $\phi_{\iota}$ is a trace for each $\iota$ and that $\phi$ is faithful if $\phi_{\iota}$ is faithful for each $\iota$.

We now describe Avitzour's result [1]. Let $A$ and $B$ be unital $C^{*}$-algebras with states $\phi$ and $\psi$, respectively. If there are unitaries $u, v \in \operatorname{ker} \phi$ and $w \in$ ker $\psi$ such that $\phi\left(u^{*} v\right)=0$ and $\phi$ and $\psi$ are invariant under conjugation by $u$ and $w$, respectively, then the reduced free product $C^{*}$-algebra $(A * B, \phi * \psi)$ is simple. Furthermore, if $\phi$ and $\psi$ are tracical states, $\phi * \psi$ is a unique tracical state. Let $C[0,1]$ be the $C^{*}$-algebra of all continuous functions on the unit interval $[0,1]$ and let $\mu$ be the tracial state on $C[0,1]$ given by integration with respect to the Lebesgue measure. We denote by $\left(\mathcal{A}_{n}, \mu_{n}\right)$ the reduced free product $(C[0,1], \mu) * \cdots *(C[0,1], \mu)$ of $n$ copies of $C[0,1]$ with respect to the tracial state $\mu$.

Proposition 2.1. For any integer $n \geq 2$, the reduced free product $\left(\mathcal{A}_{n}, \mu_{n}\right)$ is simple and exact. Moreover, $\mathcal{A}_{n}$ has a unique tracial state and stable rank 1.

Proof. To prove this theorem, we will use Avitzour's result [1] described as above. To do this, we decomposition $\left(\mathcal{A}_{n}, \mu_{n}\right)$ as $\left(\mathcal{A}_{1}, \mu_{1}\right) *\left(\mathcal{A}_{n-1}, \mu_{n-1}\right)$. We only have to show that Avitzour's conditions hold. That is, we will show the existence of unitaries $u, v \in \operatorname{ker} \mu_{1}$ and $w \in \operatorname{ker} \mu_{n-1}$ such that $\mu_{1}\left(u^{*} v\right)=0$ and $\mu_{1}$ and $\mu_{n-1}$ are invariant under conjugation by $u$ and $w$, respectively. We take continuous functions $u$ and $v$ on $[0,1]$ as follows: $u(t)=e^{2 \pi i t}$ and $v(t)=e^{4 \pi i t}$. We can identify $\mathcal{A}_{1}$ with the first component of $\mathcal{A}_{n-1}$. Let $w \in \mathcal{A}_{n-1}$ be the image of $u$ in the first component of $\mathcal{A}_{n-1}$. It is not hard to see that $\mu_{1}(u)=\mu_{1}(v)=\mu_{n-1}(w)=0$ and $\mu_{1}\left(u^{*} v\right)=0$. Moreover, we have that

$$
\begin{aligned}
\mu_{1}\left(u^{*} a u\right) & =\mu_{1}(a) \quad \text { for } a \in \mathcal{A}_{1}, \\
\mu_{n-1}\left(w^{*} b w\right) & =\mu_{n-1}(b) \quad \text { for } b \in \mathcal{A}_{n-1} .
\end{aligned}
$$

By Avitzour's result, $\left(\mathcal{A}_{n}, \mu_{n}\right)$ is simple and has a unique trace. It follows from [4] that each $\mathcal{A}_{n}$ has stable rank 1. Exactness also follows from the Dykema's result that every reduced amalgamated free product of exact separable $C^{*}$ algebras is exact.

Corollary 2.2. For any integer $n, m \geq 2, \mathcal{A}_{n} \otimes_{\min } \mathcal{A}_{m}$ is simple and has a unique trace where the tensor product is spatial.

Proof. The simplicity follows from the fact that the spatial tensor product of simple $C^{*}$-algebras is simple. Furthermore, this algebra has a unique trace.

Popa [11] presented a method of computing the von Neumann algebra generated by the normalizer of certain subalgebras in some $\mathrm{II}_{1}$-factors. Using this, 
he showed that $L\left(\mathbb{F}_{S}\right)$ has no Cartan subalgebras and is not a tensor product of $\mathrm{II}_{1}$-factors where $\mathbb{F}_{S}$ is a free group with generators in an uncountable set $S$. Using Voiculescu's free entropy theory, Ge [6] has proved that free group factors $L\left(\mathbb{F}_{n}\right)(n \geq 2)$ are prime, that is, $L\left(\mathbb{F}_{n}\right)$ are not isomorphic to the tensor product of any two factors of type $\mathrm{II}_{1}$. The existence of prime $\mathrm{II}_{1}$-factors was one of longstanding questions concerning the basic structure of factors. The following result is motivated by Ge's result.

Proposition 2.3. For any integer $n \geq 2, \mathcal{A}_{n}$ is not isomorphic to $\mathcal{A}_{p} \otimes_{\min } \mathcal{A}_{q}$ $(p, q \geq 1)$ where the tensor product is spatial.

Proof. If $p$ or $q$ is 1 , then $\mathcal{A}_{p} \otimes_{\min } \mathcal{A}_{q}$ is not simple, so that $\mathcal{A}_{p} \otimes_{\min } \mathcal{A}_{q}$ is not isomorphic to $\mathcal{A}_{n}$ since each $\mathcal{A}_{n}(n \geq 2)$ is simple. Suppose that $p, q \geq 2$ and that $\mathcal{A}_{n}$ is isomorphic to $\mathcal{A}_{p} \otimes_{\min } \mathcal{A}_{q}$ for some integer $n \geq 2$. We first note that the tracial state $\mu_{n}$ is unique for each $n \geq 2$. Let $\pi_{k}$ be the GNS representation associated with a faithful normalized tracial state $\mu_{k}$ of $\mathcal{A}_{k}(k \geq$ 2 ). Then $\pi_{k}\left(\mathcal{A}_{k}\right)^{\prime \prime}$ is isomorphic to the free group factor $L\left(\mathbb{F}_{k}\right)(k \geq 2)$ (see Theorem 7.9 in [8]). The latter is not the tensor product of any two factors of type $\mathrm{II}_{1}$ (see [6] for the proof). From the uniqueness of the tracial state, it follows that this isomorphism induces an isomorphism of weak operator closures such that $\pi_{n}\left(\mathcal{A}_{n}\right)^{\prime \prime}$ is isomorphic to the von Neumann algebra tensor product $\pi_{p}\left(\mathcal{A}_{p}\right)^{\prime \prime} \bar{\otimes} \pi_{q}\left(\mathcal{A}_{q}\right)^{\prime \prime}$. However, this contradicts the fact that $L\left(\mathbb{F}_{k}\right)(k \geq 2)$ is a prime factor. Thus $\mathcal{A}_{n}$ is not isomorphic to $\mathcal{A}_{p} \otimes_{\min } \mathcal{A}_{q}$.

For each positive integer $k$, let $M_{k}$ denote the $C^{*}$-algebra of $k \times k$ matrices over $\mathbb{C}$ and let $\operatorname{tr}_{k}$ be the normalized trace on $M_{k}$. Consider the spatial tensor product $C[0,1] \otimes M_{k}$ of two $C^{*}$-algebras $C[0,1]$ and $M_{k}$ and we denote by $\tau$ the tracial state $\mu \otimes \operatorname{tr}_{k}$ on $C[0,1] \otimes M_{k}$. We denote by $\left(\mathcal{B}_{n}, \tau_{n}\right)$ the reduced free product $\left(C[0,1] \otimes M_{k}, \tau\right) * \cdots *\left(C[0,1] \otimes M_{k}, \tau\right)$ of $n$-copies of the $C^{*}$-algebra $C[0,1] \otimes M_{k}$ with respect to the tracial state $\tau$ where $\tau_{n}$ is obtained by the free product of $n$-copies of $\tau$. We will identify the $C^{*}$-algebra $C[0,1] \otimes M_{k}$ with the algebra $C\left([0,1] \rightarrow M_{k}\right)$ of continuous functions from $[0,1]$ to $M_{k}$.

Corollary 2.4. Let $m, n \geq 2$ be positive integers.

(1) $\left(\mathcal{B}_{n}, \tau_{n}\right)$ is simple and has a unique tracial state.

(2) $\mathcal{B}_{m} \otimes_{\min } \mathcal{B}_{n}$ is simple and has a unique trace.

(3) For each integer $q \geq 2, \mathcal{B}_{q}$ is not isomorphic to $\mathcal{B}_{m} \otimes_{\min } \mathcal{B}_{n}$.

Moreover, each $\mathcal{B}_{n}$ has stable rank 1 and is exact.

Proof. To prove (1), we first decompose $\left(\mathcal{B}_{n}, \tau_{n}\right)$ as $\left(\mathcal{B}_{1}, \tau\right) *\left(\mathcal{B}_{n-1}, \tau_{n-1}\right)$. We define two functions $f$ and $g$ in $C\left([0,1] \rightarrow M_{k}\right)$ as follows:

$$
f(t)=\operatorname{diag}\left(e^{2 \pi i t}, \ldots, e^{2 \pi i t}\right) \text { and } g(t)=\operatorname{diag}\left(e^{4 \pi i t}, \ldots, e^{4 \pi i t}\right),
$$

that is, $f(t)$ and $g(t)$ are diagonal $k \times k$ matrices with entries $e^{2 \pi i t}$ and $e^{4 \pi i t}$, respectively. Identifying $\mathcal{B}_{1}$ with the first factor of $\mathcal{B}_{n-1}$, we let $h \in \mathcal{B}_{n-1}$ be 
the image of $f$ in the first factor of $\mathcal{B}_{n-1}$. It is not hard to see that $\tau(f)=$ $\tau(g)=\tau_{n-1}(h)=0$ and $\tau\left(f^{*} g\right)=0$. Moreover, we have that

$$
\begin{aligned}
\tau\left(f^{*} b f\right) & =\tau(b) \quad \text { for } b \in \mathcal{B}_{1}, \\
\tau_{n-1}\left(h^{*} c h\right) & =\tau_{n-1}(c) \quad \text { for } c \in \mathcal{B}_{n-1} .
\end{aligned}
$$

Hence $\left(\mathcal{B}_{n}, \tau_{n}\right)$ is simple and has a unique trace.

(2) It follows from (1) and the simplicity of the spatial tensor product of simple $C^{*}$-algebras.

(3) We still denote by $\mu$ the tracial state on $L^{\infty}[0,1]$ induced by the Lebesgue integration and also denote by $\tau$ the trace $\mu \otimes \operatorname{tr}_{k}$ on $L^{\infty}[0,1] \otimes M_{k}$. Since the free product $M_{k}\left(L^{\infty}[0,1]\right) * \cdots * M_{k}\left(L^{\infty}[0,1]\right)$ of $n$ copies of $M_{k}\left(L^{\infty}[0,1]\right)$ with respect to the trace $\tau$ is isomorphic to $L\left(\mathbb{F}_{n}\right)$, the result follows from the uniqueness of the trace and the primality of the free group factors. By the Dykema's results [4], the remains are true.

In Corollary 2.4, we showed that each $\mathcal{B}_{q}(q \geq 2)$ is not isomorphic to tensor products of two or more of $\mathcal{B}_{m}$ 's. It follows from [10] that minimal tensor products of different lengths are non-isomorphic like the case of free group factors. In particular, the spatial tensor product $\bigotimes_{k=1}^{p} \mathcal{B}_{n}$ is not isomorphic to the spatial tensor product $\bigotimes_{k=1}^{q} \mathcal{B}_{n}$ if $p \neq q$. A countable group $G$ is said to have the Kazhdan's property $T$ if the trivial representation $1_{G}$ is isolated in the set of all irreducible representations of $G$. Connes [2] proved that the group von Neumann algebra $L(G)$ has a countable discrete outer automorphism group if $G$ is a countable discrete i.c.c. group with the Kazhdan's property $T$. Connes and Jones have defined property $T$ for a finite von Neumann algebra. They [3] proved that a countable i.c.c. group has the Kazhdan's property $T$ if and only if the corresponding group von Neumann algebra $L(G)$ has property $T$.

Theorem 2.5. Let $G$ be a countable group with the Kazhdan's property $T$. The reduced group $C^{*}$-algebra $C_{r}^{*}(G)$ is not isomorphic to $\mathcal{A}_{n_{1}} \otimes_{\min } \cdots \otimes_{\min } \mathcal{A}_{n_{k}}$ or $\mathcal{B}_{n_{1}} \otimes_{\min } \cdots \otimes_{\min } \mathcal{B}_{n_{k}}$ for $n_{i} \geq 1$ and $k \geq 1$. In particular, $C_{r}^{*}(G)$ can not be expressed by the minimal tensor product of $C_{r}^{*}\left(\mathbb{F}_{n}\right)(n \geq 2)$ together with $C^{*}$-algebra with a unique normalized trace.

Proof. Note first that the outer automorphism group of a free product of finite von Neumann algebras is very large, at least, uncountable (see Proposition 3.5 in [7]). Since $\pi_{n_{i}}\left(\mathcal{A}_{n_{i}}\right)^{\prime \prime}$ and $\pi_{n_{i}}\left(\mathcal{B}_{n_{i}}\right)^{\prime \prime}$ have uncountable outer automorphism groups, $C_{r}^{*}(G)$ is not isomorphic to $\mathcal{A}_{n_{1}} \otimes_{\min } \cdots \otimes_{\min } \mathcal{A}_{n_{k}}$ or $\mathcal{B}_{n_{1}} \otimes_{\min } \cdots \otimes_{\min } \mathcal{B}_{n_{k}}$. To show the second part, note that the free group factor $L\left(\mathbb{F}_{n}\right)$ is isomorphic to a free product of $n$ copies of $L^{\infty}([0,1])$ with the trace given by the Lebesgue integration. It is known that the automorphism group of $L^{\infty}([0,1])$ is uncountable, in particular, non-trivial automorphisms are all outer, so that the outer automorphism group of $L\left(\mathbb{F}_{n}\right)$ is uncountable (see [7] for the detailed proof). However, the outer automorphism group $\operatorname{Out}(L(G))$ of $L(G)$ is countable. Hence we get the conclusion, that is, $C_{r}^{*}(G)$ is not the 
minimal tensor product of $C_{r}^{*}\left(\mathbb{F}_{n}\right)(n \geq 2)$ together with $C^{*}$-algebra with a unique normalized trace.

In fact, we can see that the $C^{*}$-algebra $C_{r}^{*}(G)$ in Theorem 2.5 is not isomorphic to the reduced free product of $C^{*}$-algebras with unique faithful normalized traces. Let $\Pi$ be the group of all finite permutations of positive integers $\mathbb{N}$, that is, an element $g \in \Pi$ is a one-to-one mapping of $\mathbb{N}$ onto itself which leaves all but a finite number of positive integers fixed. Then we can see that $\mathcal{A}_{p} \otimes_{\min } C_{r}^{*}(\Pi)$ and $\mathcal{A}_{q}$ are not isomorphic $(p, q \geq 1)$.

Let $\mathfrak{A}$ be the infinite $C^{*}$-minimal tensor product $\bigotimes_{k=1}^{\infty} \mathcal{A}_{0}$ (see [13] for infinite tensor products) where $\mathcal{A}_{0}=\mathcal{A}_{n}$ for some fixed $n \geq 2$ where $\mathcal{A}_{n}$ is as in Proposition 2.1. Let $\mu_{\infty}$ be the infinite product tracial state $\bigotimes_{k=1}^{\infty} \phi_{0}$ on $\mathfrak{A}$ with $\phi_{0}=\mu_{n}$. Similarly, let $\mathfrak{B}$ be the infinite $C^{*}$-minimal tensor product $\bigotimes_{k=1}^{\infty} \mathcal{B}_{0}$ with $\mathcal{B}_{0}=\mathcal{B}_{n}(n \geq 2)$ and $\tau_{\infty}$ the infinite product tracial state.

Proposition 2.6. Let $n \geq 2$ be a positive integer. The infinite $C^{*}$-minimal tensor product $\mathfrak{A}$ (respectively, $\mathfrak{B}$ ) is not isomorphic to the tensor product $\mathcal{A}_{n} \otimes_{\min } C_{r}^{*}(\Pi)$ (respectively, $\left.\mathcal{B}_{n} \otimes_{\min } C_{r}^{*}(\Pi)\right)$.

Proof. Since the proof for $\mathfrak{A}$ is similar to one for $\mathfrak{B}$, we will only show the case for $\mathfrak{A}$. Suppose that $\mathfrak{A}$ is isomorphic to $\mathcal{A}_{n} \otimes_{\min } C_{r}^{*}(\Pi)$. Let $\pi_{\infty}$ be the GNS representation associated with a faithful tracial state $\mu_{\infty}$ and let $\tau$ be a normalized faithful trace on $C_{r}^{*}(\Pi)$. Denote by $\pi_{1}$ (respectively, $\pi_{2}$ ) the GNS representation associated with faithful normalized traces $\mu_{n}$ (respectively, $\tau$ ) on $\mathcal{A}_{n}$ (respectively, $C_{r}^{*}(\Pi)$ ). From the uniqueness of the tracial states, it follows that this isomorphism induces an isomorphism of weak operator closures such that $\pi_{\infty}(\mathfrak{A})^{\prime \prime}$ is isomorphic to the von Neumann algebra tensor product $\pi_{1}\left(\mathcal{A}_{n}\right)^{\prime \prime} \bar{\otimes} \pi_{2}\left(C_{r}^{*}(\Pi)\right)^{\prime \prime}$. We know that the former is isomorphic to the $W^{*}$ infinite tensor product $\bar{\bigotimes}_{k=1}^{\infty} L\left(\mathbb{F}_{n}\right)$, but the latter is isomorphic to $L\left(\mathbb{F}_{n}\right) \bar{\otimes} \mathcal{R}$ where $\mathcal{R}$ is the hyperfinite $\mathrm{II}_{1}$-factor. However, $\bar{\bigotimes}_{k=1}^{\infty} L\left(\mathbb{F}_{n}\right)$ and $L\left(\mathbb{F}_{n}\right) \bar{\otimes} \mathcal{R}$ are not isomorphic [10] since while the former is asymptotically abelian, the latter is not. Therefore, $\mathfrak{A}$ is not isomorphic to $\mathcal{A}_{n} \otimes_{\min } C_{r}^{*}(\Pi)$.

\section{On simplicity and freeness of free product factors}

When we study free products of $C^{*}$-algebras and von Neumann algebras, the first thing we want to understand is the freeness in the free product. In this section we will discuss the freeness in free product factors. Before the discussion, we will give an elementary proof of Dykema result [4] of free product of $C^{*}$-algebras. The following proposition may be known, but we do not know a reference.

Proposition 3.1. Suppose that $\mathcal{F}_{1}$ and $\mathcal{F}_{2}$ are subsets of finite von Neumann algebras $\mathcal{M}_{1}$ and $\mathcal{M}_{2}$, respectively, such that $\mathcal{F}_{1} \cup\{I\}$ and $\mathcal{F}_{2} \cup\{I\}$ are orthogonal (or orthonormal) bases for $L^{2}\left(\mathcal{M}_{1}, \tau_{1}\right)$ and $L^{2}\left(\mathcal{M}_{2}, \tau_{2}\right)$, respectively. Then $\left(\mathcal{F}_{1} * \mathcal{F}_{2}\right) \cup\{I\}$ is an orthogonal (or orthonormal) basis of $L^{2}\left(\mathcal{M}_{1} * \mathcal{M}_{2}, \tau\right)$ where $\tau=\tau_{1} * \tau_{2}$. 
Proof. We first show that $\left(\mathcal{F}_{1} * \mathcal{F}_{2}\right) \cup\{I\}$ is an orthogonal subset of $L^{2}\left(\mathcal{M}_{1} *\right.$ $\left.\mathcal{M}_{2}, \tau\right)$. For any two elements $x$ and $y$ in $\mathcal{F}_{1} * \mathcal{F}_{2}$, suppose that $x=x_{1} x_{2} \cdots x_{m}$ and $y=y_{1} y_{2} \cdots y_{n}$ with $x_{i}$ and $y_{j}$ in $\mathcal{F}_{1} \cup \mathcal{F}_{2}$. Since elements in $\mathcal{F}_{1}$ and $\mathcal{F}_{2}$ are orthogonal to $I$, we have that

$$
\tau\left(x_{i}\right)=\tau\left(y_{j}\right)=0 \quad \text { for } i=1, \ldots, m \text { and } j=1, \ldots, n .
$$

Hence we obtain that $\langle x, I\rangle=\tau(x)=0$, which implies that $\mathcal{F}_{1} * \mathcal{F}_{2}$ is orthogonal to $I$. We want to show that $\langle x, y\rangle=0$ for $x \neq y$. Without loss of generality, we may assume that $x_{1} \in \mathcal{F}_{1}$. If $x_{1} \neq y_{1}$, then either $y_{1}$ lies in $\mathcal{F}_{1}$, or in $\mathcal{F}_{2}$. In the first case, $x_{1}$ and $y_{1}$ lie in $\mathcal{F}_{1}$ and thus are orthogonal in $L^{2}\left(\mathcal{M}_{1}, \tau_{1}\right)$, that is, $\tau\left(y_{1}^{*} x_{1}\right)=\left\langle x_{1}, y_{1}\right\rangle=0$. By the definition of $\mathcal{F}_{1} * \mathcal{F}_{2}$, we know that $x_{2}$ and $y_{2}$ lie in $\mathcal{F}_{2}\left(\subseteq \mathcal{M}_{2}\right)$. Then $y^{*} x=y_{n}^{*} \cdots y_{2}^{*}\left(y_{1}^{*} x_{1}\right) x_{2} \cdots x_{m}$ is a monomial of length $n+m-1$. In the other case (when $y_{1} \in \mathcal{F}_{2}$ ) $y^{*} x$ is also a monomial of length $n+m$, so that $\langle x, y\rangle=\tau\left(y^{*} x\right)=0$.

If $x_{1}=y_{1}$, then there is $k \geq 1$ such that $x_{j}=y_{j}$ for $1 \leq j \leq k$ and $x_{k+1} \neq y_{k+1}$. Since $x \neq y$, we may assume that $n \geq m$ and $n>k$. Then $x_{k+1}$ and $y_{k+1}$ belong to the same set $\mathcal{F}_{1}$ or $\mathcal{F}_{2}$, and $\tau\left(y_{k+1}^{*} x_{k+1}\right)=0$. Now we have that

$$
\begin{aligned}
\langle x, y\rangle= & \tau\left(y^{*} x\right)=\tau\left(y_{n}^{*} \cdots y_{2}^{*} y_{1}^{*} x_{1} x_{2} \cdots x_{m}\right) \\
= & \tau\left(y_{n}^{*} \cdots y_{k+1}^{*} x_{k}^{*} \cdots x_{2}^{*}\left(x_{1}^{*} x_{1}-\tau\left(x_{1}^{*} x_{1}\right) I\right) x_{2} \cdots x_{k} x_{k+1} \cdots x_{m}\right) \\
& +\tau\left(x_{1}^{*} x_{1}\right) \tau\left(y_{n}^{*} \cdots y_{k+1}^{*} x_{k}^{*} \cdots x_{2}^{*} x_{2} \cdots x_{k} x_{k+1} \cdots x_{m}\right) \\
= & \tau\left(x_{1}^{*} x_{1}\right) \tau\left(y_{n}^{*} \cdots y_{k+1}^{*} x_{k}^{*} \cdots x_{2}^{*} x_{2} \cdots x_{k} x_{k+1} \cdots x_{m}\right) \\
\cdots & \\
= & \tau\left(x_{1}^{*} x_{1}\right) \cdots \tau\left(x_{k}^{*} x_{k}\right) \tau\left(y_{n}^{*} \cdots y_{k+1}^{*} x_{k+1} \cdots x_{m}\right) \\
= & \tau\left(x_{1}^{*} x_{1}\right) \cdots \tau\left(x_{k}^{*} x_{k}\right) \tau\left(y_{n}^{*} \cdots y_{k+2}^{*}\left(y_{k+1}^{*} x_{k+1}\right) x_{k+2} \cdots x_{m}\right) \\
= & 0 .
\end{aligned}
$$

Therefore, $x$ is orthogonal to $y$. From the above computation, we see that if $\tau\left(x_{i}^{*} x_{i}\right)=1$, then

$$
\tau\left(x^{*} x\right)=\tau\left(x_{1}^{*} x_{1}\right) \cdots \tau\left(x_{m}^{*} x_{m}\right)=1 .
$$

Thus when $\mathcal{F}_{1}$ and $\mathcal{F}_{2}$ consist of unit vectors, then so does $\mathcal{F}_{1} * \mathcal{F}_{2}$.

Now we show that the linear span of $\left(\mathcal{F}_{1} * \mathcal{F}_{2}\right) \cup\{I\}$ is dense in $L^{2}\left(\mathcal{M}_{1} *\right.$ $\left.\mathcal{M}_{2}, \tau\right)$. To see this, we only need to show that finite linear combinations of elements in $\left(\mathcal{F}_{1} * \mathcal{F}_{2}\right) \cup\{I\}$ approximate any monomial $z_{1} z_{2} \cdots z_{n}$ in $\mathcal{M}_{1} * \mathcal{M}_{2}$ arbitrarily close in the trace norm. This follows from the inequality

$$
\|A B C\|_{2} \leq\|A\|\|B\|_{2}\|C\|
$$

and the assumption that $\mathcal{F}_{1} \cup\{I\}$ and $\mathcal{F}_{2} \cup\{I\}$ are orthogonal bases where $\|B\|_{2}=\tau\left(B^{*} B\right)^{\frac{1}{2}}$ means the trace norm of $B$.

Given a finite von Neumann algebra $\mathcal{M}$ with a finite faithful trace $\tau$, a Haar unitary (with respect to $\tau$ ) is a unitary, $u \in \mathcal{M}$, such that $\tau\left(u^{n}\right)=0$ for every 
non-zero integer $n$. If $u$ is a Haar unitary in a $*$-probability space, then the normalized Lebesgue measure on the circle serves as a $*$-distribution for $u$.

Let $\mathcal{M}_{1}$ and $\mathcal{M}_{2}$ be finite von Neumann algebras with finite faithful traces $\tau_{1}$ and $\tau_{2}$, respectively. Let $\mathcal{A}$ be a unital separable $C^{*}$-subalgebra of $\mathcal{M}_{1}$ which is ultraweakly dense in $\mathcal{M}_{1}$ and contains a Haar unitary $u$. Let $v$ be a Haar unitary in $\mathcal{M}_{2}$ with respect to $\tau_{2}$. We denote by $\mathfrak{A}$ the $C^{*}$-subalgebra in the free product $\left(\mathcal{M}_{1} * \mathcal{M}_{2}, \tau\right)$ generated by $\mathcal{A}$ and $v$ where $\tau$ is the trace given as in Proposition 3.1. We define sets $\mathcal{F}_{i}(i=1,2)$ as follows:

$$
\begin{aligned}
& \mathcal{F}_{1}=\left\{v^{n_{0}} a_{1} v^{n_{1}} \cdots a_{k} v^{n_{k}}: a_{j} \in \mathcal{A} \text { with } \tau_{1}\left(a_{j}\right)=0, k \geq 1\right. \text { and } \\
& \left.n_{j} \neq 0(1 \leq j \leq k-1)\right\}, \\
& \mathcal{F}_{2}=\left\{v^{n}: n \in \mathbb{Z} \backslash\{0\}\right\} .
\end{aligned}
$$

Note that $n_{0}$ and $n_{k}$ may be zero. Let $\mathcal{F}_{0}=\mathcal{F}_{1} \cup \mathcal{F}_{2}$ and $\mathcal{F}=\mathcal{F}_{0} \cup\{I\}$. We denote still by $\tau$ the restriction of the trace on $\mathcal{M}_{1} * \mathcal{M}_{2}$ to $\mathfrak{A}$. All finite linear combinations of elements in $\mathcal{F}$, denoted by $\mathbb{C} \mathcal{F}$, form a norm dense $*$-subalgebra of $\mathfrak{A}$. The length $\ell(x)$ of an element $x$ in $\mathcal{F}$ is defined by

$$
\ell(x)= \begin{cases}\left|n_{0}\right|+\cdots+\left|n_{k}\right| & \text { if } x=v^{n_{0}} a_{1} v^{n_{1}} \cdots a_{k} v^{n_{k}} \\ 0 & \text { if } x=I .\end{cases}
$$

The following theorem which extends Powers' result to a free product of finite von Neumann algebras are the same as that of Dykema [4], but we will give a proof by a direct variation on Powers' proof for the reduced $C^{*}$-algebra of non-abelian free groups.

Theorem 3.2. Let $\mathfrak{A}$ be a $C^{*}$-subalgebra in $\mathcal{M}_{1} * \mathcal{M}_{2}$ generated by $\mathcal{A}$ and $v$ where $\mathcal{M}_{1}, \mathcal{M}_{2}, \mathcal{A}$ and $v$ are same as above. Then the $C^{*}$-algebra $\mathfrak{A}$ is simple and has a unique trace.

Proof. Suppose that $\mathfrak{J}$ is a non-zero two-sided ideal in $\mathfrak{A}$. We can choose a nonzero positive element $x$ in $\mathfrak{J}$ with $\tau(x) \neq 0$. Multiplying $x$ by some constant $\lambda \in \mathbb{C}$ if necessary, we may assume that $\tau(x)=1$. From the Kaplansky's density theorem, we know that for any $\epsilon>0$ there is a self-adjoint element $y$ in $\mathbb{C} \mathcal{F}$ such that

$$
\|x-y\|<\epsilon \text { and }\|y\| \leq\|x\| .
$$

We also assume that $\tau(y)=1$ so that we can write

$$
y=I+\sum_{j=1}^{n} \lambda_{j} y_{j} \quad \text { for some } \lambda_{j} \in \mathbb{C} \text { and } y_{j} \in \mathcal{F}_{0}(j=1, \ldots, n)
$$

since traces of all elements in $\mathcal{F}_{0}$ are zero.

Let $m_{0}-1$ be the maximum of lengths of $y_{1}, \ldots, y_{n}$, that is,

$$
m_{0}-1=\max \left\{\ell\left(y_{j}\right): 1 \leq j \leq n\right\} .
$$

Then the elements $v^{-m_{0}} y_{j} v^{m_{0}}$ begin and end with a nonzero power of $v$ for all $j=1, \ldots, n$. Let $\mathcal{C}$ be the set of elements in $\mathcal{F}_{0}$ which begins by $v^{m_{0}}$ (followed 
by a non-trivial element in $\mathcal{A}$, or by nothing at all) and let $\mathcal{D}=\mathcal{F}-\mathcal{C}$. Then we see that $\mathcal{C}$ and $\mathcal{D}$ are orthogonal. From above argument, we also see that $y_{j} \mathcal{C}$ and $\mathcal{C}$ are orthogonal for each $j=1, \ldots, n$. Since $\mathcal{D}=\mathcal{F}-\mathcal{C}$, one can see that $u^{i} v^{-m_{0}} \mathcal{D}$ and $u^{j} v^{-m_{0}} \mathcal{D}$ are orthogonal whenever $i \neq j$. Since $\mathcal{A}$ is ultraweakly dense in $\mathcal{M}_{1}$, we have that $L^{2}(\mathfrak{A}, \tau)=L^{2}\left(\mathcal{M}_{1} *\{v\}^{\prime \prime}, \tau\right)$.

Let $\mathcal{K}$ be the closed subspace spanned by $\mathcal{D}$ and $P$ the projection from $L^{2}(\mathfrak{A}, \tau)$ onto $\mathcal{K}$. For each positive integer $j$, we define $w_{j}$ and $Q_{j}$ by

$$
w_{j}=u^{j} v^{-m_{0}} \text { and } Q_{j}=w_{j} P w_{j}^{-1} \text {. }
$$

Since $w_{i} \mathcal{D}$ and $w_{j} \mathcal{D}$ are orthogonal for $i \neq j$, we see that $Q_{1}, Q_{2}, \ldots$ are pairwise orthogonal projections. Moreover, $y_{j} \mathcal{C}$ and $\mathcal{C}$ are also orthogonal. Hence we have that

$$
(I-P) y_{j}(I-P)=0 \text { for } j=1, \ldots, n .
$$

It follows from the definition of $Q_{j}$ that $I-P=w_{j}^{-1}\left(I-Q_{j}\right) w_{j}$. Since

$$
(I-P) y_{j}(I-P)=0 \text { for each } 1 \leq j \leq n,
$$

we obtain that $(I-P)\left(\sum_{j=1}^{n} \lambda_{j} y_{j}\right)(I-P)=0$. Therefore, we get the equality

$$
0=\left(I-Q_{j}\right) w_{j}\left(\sum_{j=1}^{n} \lambda_{j} y_{j}\right) w_{j}^{-1}\left(I-Q_{j}\right) .
$$

Now we prove that the inequality

$$
\left\|\frac{1}{k} \sum_{j=1}^{k} w_{j}\left(\sum_{i=1}^{n} \lambda_{i} y_{i}\right) w_{j}^{-1}\right\| \leq \frac{2}{\sqrt{k}}\left\|\sum_{i=1}^{n} \lambda_{i} y_{i}\right\|
$$

holds for each positive integer $k \geq 1$. If $\xi \in L^{2}(\mathfrak{A}, \tau)$ is a unit vector, we have that

$$
\begin{aligned}
& \left|\left\langle\frac{1}{k} \sum_{j=1}^{k} w_{j}\left(\sum_{i=1}^{n} \lambda_{i} y_{i}\right) w_{j}^{-1} \xi, \xi\right\rangle\right| \\
\leq & \frac{1}{k} \sum_{j=1}^{k}\left|\left\langle w_{j}\left(\sum_{i=1}^{n} \lambda_{i} y_{i}\right) w_{j}^{-1} \xi, \xi\right\rangle\right| \\
= & \frac{1}{k} \sum_{j=1}^{k}\left|\left\langle\left(I-Q_{j}+Q_{j}\right) w_{j}\left(\sum_{i=1}^{n} \lambda_{i} y_{i}\right) w_{j}^{-1}\left(I-Q_{j}+Q_{j}\right) \xi, \xi\right\rangle\right| \mid \\
\leq & \frac{1}{k} \sum_{j=1}^{k}\left\{\left|\left\langle\left(I-Q_{j}\right) w_{j}\left(\sum_{i=1}^{n} \lambda_{i} y_{i}\right) w_{j}^{-1}\left(I-Q_{j}\right) \xi, \xi\right\rangle\right|\right. \\
& \left.+\left|\left\langle\left(I-Q_{j}\right) w_{j}\left(\sum_{i=1}^{n} \lambda_{i} y_{i}\right) w_{j}^{-1} Q_{j} \xi, \xi\right\rangle\right|+\left|\left\langle Q_{j} w_{j}\left(\sum_{i=1}^{n} \lambda_{i} y_{i}\right) w_{j}^{-1} \xi, \xi\right\rangle\right|\right\}
\end{aligned}
$$




$$
\begin{aligned}
& \leq \frac{1}{k} \sum_{j=1}^{k}\left\{\left\|\left(I-Q_{j}\right) w_{j}\left(\sum_{i=1}^{n} \lambda_{i} y_{i}\right) w_{j}^{-1}\right\|\left\|Q_{j} \xi\right\|+\left\|w_{j}\left(\sum_{i=1}^{n} \lambda_{i} y_{i}\right) w_{j}^{-1}\right\|\left\|Q_{j} \xi\right\|\right\} \\
& \leq \frac{1}{k} \sum_{j=1}^{k}\left\|\sum_{i=1}^{n} \lambda_{i} y_{i}\right\|\left(\left\|Q_{j} \xi\right\|+\left\|Q_{j} \xi\right\|\right) \\
& \leq \frac{2}{\sqrt{k}}\left\|\sum_{i=1}^{n} \lambda_{i} y_{i}\right\| .
\end{aligned}
$$

Since $y$ is a self-adjoint element, $y-I=\sum_{i=1}^{n} \lambda_{i} y_{i}$ is also a self-adjoint element in $\mathbb{C} \mathcal{F}$. Hence get the inequality (1).

Next we will show that

$$
\left\|I-\frac{1}{k} \sum_{j=1}^{k} w_{j} x w_{j}^{-1}\right\| \leq \frac{2}{\sqrt{k}}\|x\|+\epsilon
$$

for each integer $k \geq 1$. Using the triangle inequality, we have

$$
\begin{aligned}
& \left\|I-\frac{1}{k} \sum_{j=1}^{k} w_{j} x w_{j}^{-1}\right\| \\
\leq & \left\|I-\frac{1}{k} \sum_{j=1}^{k} w_{j} y w_{j}^{-1}\right\|+\left\|\frac{1}{k} \sum_{j=1}^{k} w_{j} y w_{j}^{-1}-\frac{1}{k} \sum_{j=1}^{k} w_{j} x w_{j}^{-1}\right\| \\
= & \left\|\frac{1}{k} \sum_{j=1}^{k} w_{j}\left(\sum_{i=1}^{n} \lambda_{i} y_{i}\right) w_{j}^{-1}\right\|+\|y-x\| \\
\leq & \frac{2}{\sqrt{k}}\left\|\sum_{i=1}^{n} \lambda_{i} y_{i}\right\|+\epsilon \\
\leq & \frac{2}{\sqrt{k}}\|x\|+\epsilon .
\end{aligned}
$$

By the inequality (2) we have the inequality

$$
\left\|I-\frac{1}{k} \sum_{j=1}^{k} w_{j} x w_{j}^{-1}\right\|<1 \text { for sufficiently large } k \text {. }
$$

This inequality implies that $b=\frac{1}{k} \sum_{j=1}^{k} w_{j} x w_{j}^{-1}$ is invertible in $\mathfrak{A}$. However, the element $x$ belongs to $\mathfrak{J}$ and $\mathfrak{J}$ is a two-sided ideal, so that $b$ lies in $\mathfrak{J}$. Since the ideal $\mathfrak{J}$ contains an invertible element, the closed ideal $\mathfrak{J}$ contains $\mathfrak{A}$. Therefore, the $C^{*}$-algebra $\mathfrak{A}$ is simple.

To show that $\mathfrak{A}$ has a unique trace, let $\tau^{\prime}$ be any normalized trace on $\mathfrak{A}$. Suppose that $x \in \mathfrak{A}$ and $\epsilon>0$ are given. Then it follows from the above proof 
that there are unitaries $w_{j} \in \mathcal{F}$ and $\lambda_{j} \in \mathbb{C}(j=1, \ldots, n)$ with $\sum_{j} \lambda_{j}=1$ such that

$$
\left\|\tau(x) I-\sum_{j=1}^{n} \lambda_{j} w_{j} x w_{j}^{*}\right\|<\epsilon .
$$

Hence, we have that

$$
\begin{aligned}
\left|\tau^{\prime}\left(\tau(x) I-\sum_{j=1}^{n} \lambda_{j} w_{j} x w_{j}^{*}\right)\right| & =\left|\tau(x)-\sum_{j=1}^{n} \lambda_{j} \tau^{\prime}\left(w_{j} x w_{j}^{*}\right)\right| \\
& =\left|\tau(x)-\tau^{\prime}(x)\right|<\epsilon .
\end{aligned}
$$

Since $\epsilon>0$ is arbitrary, we have that $\tau(x)=\tau^{\prime}(x)$ for every $x \in \mathfrak{A}$. Hence $\mathfrak{A}$ has a unique trace.

Suppose that $\mathcal{M}$ is a finite von Neumann algebra with a faithful normal trace $\tau$. Let $\operatorname{Aut}(\mathcal{M})$ denote the group of the trace preserving $*$-automorphisms of $\mathcal{M}$ and $\operatorname{Int}(\mathcal{M})$ the group of inner automorphisms of $\mathcal{M}$. Let $\operatorname{Out}(\mathcal{M})$ be the quotient $\operatorname{Aut}(\mathcal{M}) / \operatorname{Int}(\mathcal{M})$. When $\mathcal{M}$ is a factor of type $\mathrm{II}_{1}$, there is a unique normalized trace on $\mathcal{M}$ and all *-automorphisms always preserve the trace. The following proposition shows that any pair of automorphisms on finite von Neumann algebras $\mathcal{M}_{1}$ and $\mathcal{M}_{2}$ implement in a natural way an automorphism on the free product $\mathcal{M}_{1} * \mathcal{M}_{2}$. It may be well-known, but we cannot find a reference so that we give a more detailed proof for the convenience of a reader.

Proposition 3.3. Let $\mathcal{M}_{1}$ and $\mathcal{M}_{2}$ be finite von Neumann algebras with normalized faithful normal traces $\tau_{1}$ and $\tau_{2}$, respectively. For any $\alpha_{1}$ in $\operatorname{Aut}\left(\mathcal{M}_{1}\right)$ and $\alpha_{2}$ in $\operatorname{Aut}\left(\mathcal{M}_{2}\right)$, there is a unique trace preserving *-automorphism, denoted by $\alpha_{1} * \alpha_{2}$, of the free product $\mathcal{M}_{1} * \mathcal{M}_{2}$ such that $\left.\alpha_{1} * \alpha_{2}\right|_{\mathcal{M}_{1}}=\alpha_{1}$ and $\left.\alpha_{1} * \alpha_{2}\right|_{\mathcal{M}_{2}}=\alpha_{2}$.

Proof. We define a mapping $\alpha$ of the algebraic free product $\mathcal{M}_{1} *_{a} \mathcal{M}_{2}$ into $\mathcal{M}_{1} *_{a} \mathcal{M}_{2}$ by

$$
\alpha(I)=I \text { and } \alpha\left(x_{1} x_{2} \cdots x_{n}\right)=\alpha_{i_{1}}\left(x_{1}\right) \alpha_{i_{2}}\left(x_{2}\right) \cdots \alpha_{i_{n}}\left(x_{n}\right),
$$

where $x_{1} x_{2} \cdots x_{n}$ is a monomial in the algebraic free product $\mathcal{M}_{1} *_{a} \mathcal{M}_{2}$ such that $x_{j} \in \mathcal{M}_{i_{j}}, i_{1} \neq i_{2} \neq \cdots \neq i_{n}$ and $i_{j} \in\{1,2\}$. Extend linearly to the algebraic free product $\mathcal{M}_{1} *_{a} \mathcal{M}_{2}$. Since $\alpha_{1}$ and $\alpha_{2}$ are trace preserving automorphisms, the mapping $\alpha$ is also trace preserving on $\mathcal{M}_{1} *_{a} \mathcal{M}_{2}$ and maps $\mathcal{M}_{1} *_{a} \mathcal{M}_{2}$ onto $\alpha_{1}\left(\mathcal{M}_{1}\right) *_{a} \alpha_{2}\left(\mathcal{M}_{2}\right)\left(=\mathcal{M}_{1} *_{a} \mathcal{M}_{2}\right)$.

Moreover, we have that for any monomial $x_{1} x_{2} \cdots x_{n}$ in $\mathcal{M}_{1} *_{a} \mathcal{M}_{2}$,

$$
\begin{aligned}
\alpha\left(\left(x_{1} x_{2} \cdots x_{n}\right)^{*}\right) & =\alpha\left(x_{n}^{*} \cdots x_{2}^{*} x_{1}^{*}\right) \\
& =\alpha_{i_{n}}\left(x_{n}^{*}\right) \cdots \alpha_{i_{2}}\left(x_{2}^{*}\right) \alpha_{i_{1}}\left(x_{1}^{*}\right) \\
& =\alpha_{i_{n}}\left(x_{n}\right)^{*} \cdots \alpha_{i_{2}}\left(x_{2}\right)^{*} \alpha_{i_{1}}\left(x_{1}\right)^{*} \\
& =\left(\alpha_{i_{1}}\left(x_{1}\right) \alpha_{i_{2}}\left(x_{2}\right) \cdots \alpha_{i_{n}}\left(x_{n}\right)\right)^{*} \\
& =\alpha\left(x_{1} x_{2} \cdots x_{n}\right)^{*} .
\end{aligned}
$$


It is a routine calculation to see that $\alpha(x y)=\alpha(x) \alpha(y)$ for $x$ and $y$ in $\mathcal{M}_{1} *_{a} \mathcal{M}_{2}$ and that $\alpha$ is one-to-one. Thus $\alpha$ is a trace preserving $*$-automorphism of $\mathcal{M}_{1} * a$ $\mathcal{M}_{2}$. Because the algebraic free product $\mathcal{M}_{1} *{ }_{a} \mathcal{M}_{2}$ is dense in $L^{2}\left(\mathcal{M}_{1} * \mathcal{M}_{2}, \tau\right)$ where $\tau=\tau_{1} * \tau_{2}, \alpha$ extends to a unitary operator $u$ on the Hilbert space $L^{2}\left(\mathcal{M}_{1} * \mathcal{M}_{2}, \tau\right)$ and $u^{*}$ is the extension of $\alpha^{-1}$ (on $\left.\mathcal{M}_{1} *_{a} \mathcal{M}_{2}\right)$.

For any $x$ in $\mathcal{M}_{1} * \mathcal{M}_{2}$, let $\alpha_{1} * \alpha_{2}(x)$ be $u x u^{*}$. Then $\alpha_{1} * \alpha_{2}$ is an ultraweakly continuous $*$-isomorphism of the free product $\mathcal{M}_{1} * \mathcal{M}_{2}$ onto $u\left(\mathcal{M}_{1} * \mathcal{M}_{2}\right) u^{*}$. Let $\xi$ be the unit vector corresponding to $I$ in $\mathcal{M}_{1} * \mathcal{M}_{2}$. Since $\tau$ is the vector state induced by $\xi$ and $u(\xi)=\xi$, we see that $\alpha_{1} * \alpha_{2}$ preserves the trace $\tau$. For any $x_{1}$ in $\mathcal{M}_{1}$, we want to show that $\alpha_{1} * \alpha_{2}\left(x_{1}\right)=\alpha_{1}\left(x_{1}\right)$. For any element $x$ in $\mathcal{M}_{1} *_{a} \mathcal{M}_{2}\left(\subseteq L^{2}\left(\mathcal{M}_{1} * \mathcal{M}_{2}, \tau\right)\right)$, we know that $\alpha^{-1}(x)$ and so $x_{1} \alpha^{-1}(x)$ lie in $\mathcal{M}_{1} *_{a} \mathcal{M}_{2}$. Using the property that $\alpha$ is an automorphism, we have that

$$
\begin{aligned}
\alpha_{1} * \alpha_{2}\left(x_{1}\right)(x) & =u x_{1} u^{*}(x)=u x_{1}\left(\alpha^{-1}(x)\right) \\
& =u\left(x_{1} \alpha^{-1}(x)\right)=\alpha\left(x_{1} \alpha^{-1}(x)\right) \\
& =\alpha\left(x_{1}\right)(x)=\alpha_{1}\left(x_{1}\right)(x) .
\end{aligned}
$$

Similarly, we have that $\left.\alpha_{1} * \alpha_{2}\right|_{\mathcal{M}_{2}}=\alpha_{2}$. We know that $\mathcal{M}_{1} * \mathcal{M}_{2}$ is generated by $\mathcal{M}_{1}$ and $\mathcal{M}_{2}$ as a von Neumann algebra, so that an ultraweakly continuous automorphism of $\mathcal{M}_{1} * \mathcal{M}_{2}$ is determined by its restrictions on $\mathcal{M}_{1}$ and $\mathcal{M}_{2}$. Hence the uniqueness of $\alpha_{1} * \alpha_{2}$ follows.

If either $\alpha_{1}$ or $\alpha_{2}$ is not an identity automorphism, then we can see that $\alpha_{1} * \alpha_{2}$ is an outer automorphism on $\mathcal{M}_{1} * \mathcal{M}_{2}$. More precisely, there is an embedding of the direct product $\operatorname{Aut}\left(\mathcal{M}_{1}\right) \times \operatorname{Aut}\left(\mathcal{M}_{2}\right) \operatorname{into} \operatorname{Out}\left(\mathcal{M}_{1} * \mathcal{M}_{2}\right)$ induced by the free product of automorphisms defined in Proposition 3.3 (see [7] for more details). To discuss the freeness in a free product of von Neumann algebra, we consider the given questions in the beginning of this section: for any abelian von Neumann subalgebra $\mathcal{A}$ of a free product $\mathrm{II}_{1}$-factor $\mathcal{M}$, is there a projection $P(\neq 0, I)$ in $\mathcal{M}$ such that $\mathcal{A}$ and $P$ are free? Here free means that the von Neumann subalgebra generated by $\mathcal{A}$ and $P$ is $*$-isomorphic to the free product of $\mathcal{A}$ and the algebra generated by $P$ and $I$. It is proved by Voiculescu that $L\left(\mathbb{F}_{n}\right)(n=2,3, \ldots, \infty)$ doesn't have Cartan subalgebras. We need a lemma which was proved in [7] to prove Theorem 3.5.

Lemma 3.4 ([7]). Suppose that $\mathcal{M}_{1}$ and $\mathcal{M}_{2}$ are finite von Neumann algebras with faithful normal traces $\tau_{1}$ and $\tau_{2}$, respectively. If $\mathcal{A}$ is a non-atomic abelian von Neumann subalgebra of $\mathcal{M}_{1}$ and $w$ is a unitary operator of the free product $\mathcal{M}_{1} * \mathcal{M}_{2}$ such that $w \mathcal{A} w^{*}$ is contained in $\mathcal{M}_{1}$, then $w$ is in $\mathcal{M}_{1}$.

The following theorem shows that the above question about freeness in a free product is related to the existence of Cartan subalgebras in free product $\mathrm{II}_{1}$-factors.

Theorem 3.5. If for any abelian von Neumann subalgebra $\mathcal{A}$ in a free product $I I_{1}$-factor $\mathcal{M}$ there is a projection $P(\neq 0, I)$ in $\mathcal{M}$ such that $\mathcal{A}$ and $P$ are free, then $\mathcal{M}$ does not have any Cartan subalgebra. 
Proof. Let $\mathcal{A}$ be a maximal abelian von Neumann subalgebra of $\mathcal{M}$. By the assumption, there is a non-trivial projection $P(\neq 0, I)$ in $\mathcal{M}$ such that $\mathcal{A}$ and $P$ are free. By Lemma 3.4, we know that the normalizer of $\mathcal{A}$ in the von Neumann subalgebra generated by $\mathcal{A}$ and $P$ is contained in $\mathcal{A}$. Thus $\mathcal{A}$ can not be a Cartan subalgebra of the von Neumann algebra generated by $\mathcal{A}$ and $P$. Since any Cartan subalgebra of a von Neumann algebra is also Cartan in any subalgebra containing it, $\mathcal{A}$ can not be a Cartan subalgebra of $\mathcal{M}$. This completes the proof.

A von Neumann algebra $\mathcal{M}$ is solid if for any diffuse von Neumann subalgebra $\mathcal{A}$ in $\mathcal{M}$, the relative commutant $\mathcal{A}^{\prime} \cap \mathcal{M}$ is injective. Ozawa [9] proved that the hyperbolic group von Neumann algebra is solid, which is related to the primality of the free group factor. In particular, the free group factor is solid. Thus, we can ask the similar question for free product $\mathrm{II}_{1}$-factors: For any non-atomic injective subalgebra $\mathcal{B}$ of a free product $\mathrm{II}_{1}$-factor $\mathcal{M}$, is the relative commutant of $\mathcal{B}$ in $\mathcal{M}$ always injective? This is weaker than the solidity of a von Neumann algebra. Here we would like to answer this question in the negative. A counterexample is directly obtained from Theorem 5.2 in [5]. For the convenience of a reader, we will give the example.

Suppose that the group $G$ is the direct product of an i.c.c. (infinite conjugacy classes) amenable countable discrete group $H$ with a countable discrete group $G_{1}$. Then $L(H)$ is the hyperfinite $\mathrm{II}_{1}$-factor and we denote it by $\mathcal{R}_{2}$ which is generated by two unitary generators $U_{2}, V_{2}$ with the relation $U_{2} V_{2}=e^{2 \pi i \theta^{\prime}} V_{2} U_{2}$. Let $\mathcal{M}_{1}=\mathcal{R}_{1} * L(G)$ where $\mathcal{R}_{1}$ is generated by unitary generators $U_{1}, V_{1}$ with $U_{1} V_{1}=e^{2 \pi i \theta} V_{1} U_{1}$. If we choose $\theta^{\prime}$ such that $2 \theta^{\prime}=\theta$, then the mapping $\alpha$ given by $\alpha\left(U_{1}\right)=U_{2}$ and $\alpha\left(V_{1}\right)=V_{2}^{2}$ determines an isomorphism of $\mathcal{R}_{1}$ into $\mathcal{R}_{2}$. Let $\mathcal{N}=M_{2}(\mathbb{C}) \otimes \mathcal{M}_{1}$ and let $\mathcal{R}$ be the subalgebra of $\mathcal{N}$ consisting of all elements $\left(\begin{array}{cc}x & 0 \\ 0 & \alpha(x)\end{array}\right)$ for $x \in \mathcal{R}_{1}$. We denote by $\mathcal{M}$ the free product of $\mathcal{N}$ and $\mathcal{N}_{1}$ where $\mathcal{N}_{1}$ is any type $\mathrm{II}_{1}$-factor. By Corollary 3.2 in [7], $\mathcal{R}^{\prime} \cap \mathcal{M}$ is contained in $\mathcal{N}$, so that $\mathcal{R}^{\prime} \cap \mathcal{M}=\mathcal{R}^{\prime} \cap \mathcal{N}$. The relative commutant of $\mathcal{R}$ in $\mathcal{M}$ is not injective if $G_{1}$ is not amenable. Hence we obtain that $\mathcal{M}$ has a non-atomic injective subalgebra $\mathcal{R}$ with a non-injective relative commutant $\mathcal{R}^{\prime} \cap \mathcal{M}$ if $G_{1}$ is not amenable.

\section{References}

[1] D. Avitzour, Free products of $C^{*}$-algebras, Trans. Amer. Math. Soc. 271 (1982), no. 2, 423-435.

[2] A. Connes, A factor of type $\mathrm{II}_{1}$ with countable fundamental group, J. Operator Theory 4 (1980), no. 1, 151-153.

[3] A. Connes and V. Jones, Property $T$ for von Neumann algebras, Bull. London Math. Soc. 17 (1985), no. 1, 57-62.

[4] K. Dykema, Simplicity and the stable rank of some free product $C^{*}$-algebras, Trans. Amer. Math. Soc. 351 (1999), no. 1, 1-40.

[5] L. Ge, On maximal injective subalgebras of factors, Adv. Math. 118 (1996), no. 1, 34-70.

[6] - Applications of free entropy to finite von Neumann algebras. II, Ann. of Math. (2) 147 (1998), no. 1, 143-157. 
[7] J. Heo, On outer automorphism groups of free product factors, Internat. J. Math. 13 (2002), no. 1, 31-41.

[8] A. Nica and R. Speicher, Lectures on the Combinatorics of Free Probability, Cambridge University Press, Cambridge, 2006.

[9] N. Ozawa, Solid von Neumann algebras, Acta Math. 192 (2004), no. 1, 111-117.

[10] N. Ozawa and S. Popa, Some prime factorization results for type $\mathrm{II}_{1}$ factors, Invent. Math. 156 (2004), no. 2, 223-234.

[11] S. Popa, Orthogonal pairs of *-subalgebras in finite von Neumann algebras, J. Operator Theory 9 (1983), no. 2, 253-268.

[12] R. Powers, Simplicity of the $C^{*}$-algebra associated with the free group on two generators, Duke Math. J. 42 (1975), 151-156.

[13] S. Sakai, Asymptotically abelian $\mathrm{II}_{1}$-factors, Publ. Res. Inst. Math. Sci. Ser. A 4 (1968/1969), 299-307.

[14] A. Valette, Old and new about Kazhdan's property (T), Representations of Lie groups and quantum groups (Trento, 1993), 271-333, Pitman Res. Notes Math. Ser., 311, Longman Sci. Tech., Harlow, 1994.

[15] D. Voiculescu, Symmetries of some reduced free product $C^{*}$-algebras, Operator algebras and their connections with topology and ergodic theory, Lect. Notes in Math. 1132 (1985), 556-588.

[16] D. Voiculescu, K. Dykema, and A. Nica, Free Random Variables, CRM Monograph Series, 1. American Mathematical Society, Providence, RI, 1992.

JAESEONG HEO

Department of Mathematics

Research Institute for Natural Sciences

HANYANG UNIVERSITY

SEOUL 133-791, KOREA

E-mail address: hjs@hanyang.ac.kr

JEONG HEE KIM

Department of Mathematics

Research Institute for Natural Sciences

HANYANG UNIVERSITY

SeOul 133-791, KoreA

E-mail address: jay14@hanmail.net 